\title{
Meissner Effect (1933) Re-Explained by Gill's Electronic Theory of Magnetism (1964)
}

\author{
Avtar Singh Gill \\ avtargillmd@aol.com
}

\begin{abstract}
Curie point is reached according to Gill's electronic theory of magnetism (1964) because of the increased inter-atomic distance at a certain high temperature for a particular metal which makes it impossible for some exposed electrons of a ferromagnetic atom to latch onto the exposed protons of the next atom to cause magnetization. This Curie point could be increased by applying a stronger external magnetic field.
\end{abstract}

Meissner effect (1933) refers to the expulsion or squeezing out of an otherwise constant total magnetic flux from within the magnet to the outside on cooling of the magnet to a critical temperature as it results in less internal space. It will be shown that the concept of internal plus external magnetic flux as a constant is wrong and an alternative explanation will be presented to explain the Meissner experiment results obtained in 1933 with the help of Gill's electronic theory of magnetism (the re-explained Meissner effect).

Gill's electronic theory of magnetism shows that the reduced inter-atomic distance of the magnetized chain inside the magnetized tin cylinder on cooling will result in a greater number of electrons of one atom to latch onto the protons of the next atom and so on. This increased magnetic force of attraction between exposed electrons and protons of adjacent magnetized atoms will prevent any expulsion of the increased intra-magnetic force along its length. There is greater magnetization of the tin cylinders resultant magnetic poles at the two ends due to reduced inter-atomic distance due to cooling resulting in the development of a stronger external magnetic force around the tin cylinder, with no change in the external applied external magnetic force and this is the correct Meissner effect.

Levitation of the electron dependent north magnetic pole of a magnet in the Meissner experiment due to a dense layer of electrons on the tin surface will be addressed.

Superconductivity will be explained by super-cooling leading to a greatly reduced inter-atomic distance leading to easy flow of the free outer valence electrons as they are experiencing near zero resistance while flowing from one atom to the next. These outer free electrons in a superconducting supercooled state will experience equal force from neighboring consecutive proton masses of consecutive atoms and thus are able to move freely with zero resistance.

\section{INTRODUCTION}

A better understanding of physics leads to better applications. While dealing with magnetism, we are dealing with two forces from the two poles of a magnet. The north magnetic pole will repel another north magnetic pole and the south magnetic pole will repel another south magnetic pole and the north magnetic pole will attract the south magnetic pole. Gill's electronic theory of magnetism (1964) shows that the magnetic force is a combination of the electron dependent and proton dependent forces from the two magnetic poles and the relative motion between the very same two forces produces an electric current.

Pierre Curie while dealing with magnetism at high temperatures brought up the Curie point and Curie's law. The author has similar results with dot product equations which have been further elaborated with the application of Gill's electronic theory of magnetism.

The Walther Meissner and Robert Ochsenfeld experiment (1933) while dealing with temperatures approaching absolute zero obtained results which were explained as expulsion of magnetic flux known as the Meissner effect. Gill's electronic theory of magnetism (1964) explains the magnetic configuration of the tin cylinder atoms under the influence of an electromagnet. It is further explained why there cannot be any expulsion of internal magnetic forces although internal magnetic forces increase with cooling. The overall increase in external magnetic forces around a tin cylinder without any expulsion of magnetic flux from within is because of increased magnetization of its magnetic ends because of cooling.

A. Einstein in 1905 and Richard Feynman in 1943 expressed concern about asymmetry between the magnetic force and the electrical forces and the same is resolved with the application of Gill's electronic theory of magnetism (1964).

\section{METHOD}

Gill's electronic theory of magnetism (1964) will be summarized and its application will help in explaining the Curie point and its variation with change of applied external magnetic force. Next, Gill's 
electronic theory of magnetism (1964) will be applied to the Meissner experiment (1933) and the results will be explained. It will be shown that there is no expulsion of magnetic flux (Meissner effect), but, there is development of extra magnetic flux/force due to decreased inter-atomic distance in the tin cylinder due to cooling with all other factors remaining unchanged. It will be explained how a decreased inter-atomic distance due to cooling makes it easier for a larger number of inner electrons of one magnetized atom to latch on to the protons of the next magnetized atom and so on and we have a larger number of exposed inner electrons $(\boldsymbol{n})$ with a negative torque $(-\boldsymbol{\tau})$ at one end as the north magnetic pole and an equal larger number of exposed protons $(\boldsymbol{n})$ at the other end with a positive torque $(+\boldsymbol{\tau})$ as the south magnetic pole of the tin cylinder. This leads to extra magnetic force manifestation on the outside. It will be explained why magnetic flux cannot be pushed out of the tin surface after cooling. Superconductivity will be explained along with a presentation of stages of magnetism.

\section{GILL'S ELECTRONIC THEORY OF MAGNETISM (1964)}

Gill's electronic theory of magnetism (1964) is based on the structure of the atom and explains how the positively charged protons and the negatively charged electrons of an atom are responsible for both magnetism and electrical forces.

In the diagrams that follow in this article, we are using a simplified version of the structure of an atom with a large black proton mass and small red inner electrons. Please note that the outer free valence electrons (not shown) take part mainly in electrical current flow.

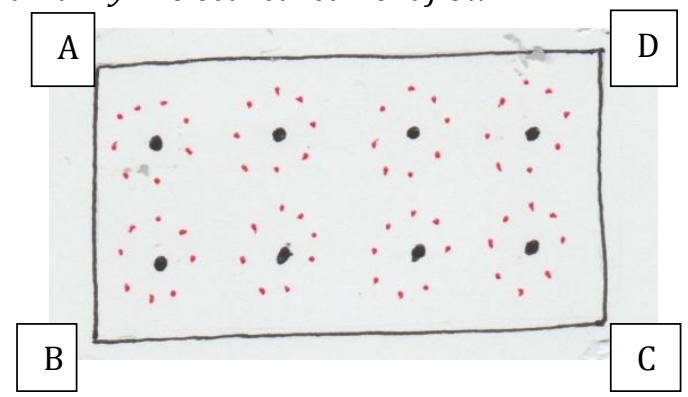

Fig1 (a): above shows neutral iron atoms in an un-magnetized state.

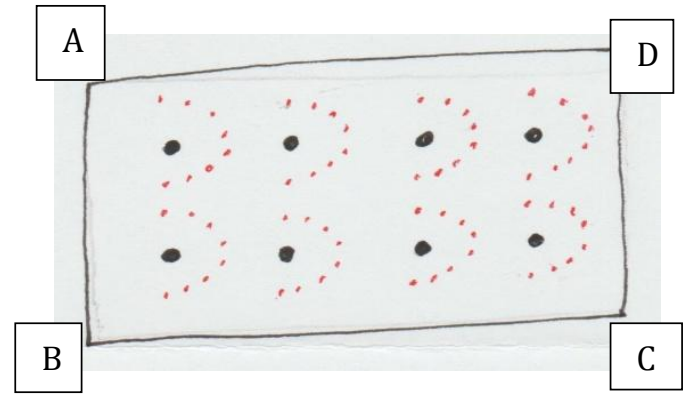

Fig1 (b): above shows the change in position of the inner electrons on magnetization.

Figure $1 \mathrm{~b}$ shows the change in position of the inner electrons on magnetization. If the displaced magnetized electrons have a negative torque $(-\boldsymbol{\tau})$, then the exposed protons at the other end of the magnetized atom will have a positive torque $(+\boldsymbol{\tau})$ and vice versa. This chain continues with the magnetic ends manifesting as the exposed negatively torqued $(-\tau)$ electron based north magnetic pole and the exposed oppositely torqued $(+\boldsymbol{\tau})$ proton based south magnetic pole of the magnet.

Gill's electronic theory of magnetism (1964) shows the neutral iron atoms in Fig1a are magnetized in Figure $1 \mathrm{~b}$ and $\mathrm{CD}$ has become the negative magnetic pole or north magnetic pole with a negatively torqued $(-\boldsymbol{\tau})$ non-moving charge - ne of the magnet and $\mathrm{AB}$ has become the positive magnetic pole or the south magnetic pole of the magnet with an opposite positively torqued $(+\boldsymbol{\tau})$ non-moving charge $+\boldsymbol{n} \boldsymbol{e}$ where $\boldsymbol{n}$ is the number of exposed inner electrons at one end and equals the number of exposed protons at the other end.

The neutral atoms in Fig 1a have become magnetized atoms in Fig $1 b$ by undergoing a change in configuration and each atom also has developed an opposing torque between its own electrons and protons to give the magnetized atoms a cork-screw effect.

In Gill's electronic theory of magnetism, magnetic force $\boldsymbol{B}$ is the force of attraction between

(1)The exposed inner electron based north magnetic pole and

(2)The exposed proton based south magnetic pole. 
The application of Gill's electronic theory of magnetism (1964) in Figure 1b shows that the magnetic force comprises of two forces (positive and negative) emanating from the proton dependent south magnetic pole and the electron dependent north magnetic pole of reconfigured magnetized atoms.

Experiment- A physicist showed me the following experiment in 1965.0n a wooden table, spread some coarse iron filings and in the middle of the iron filings, place a magnet.

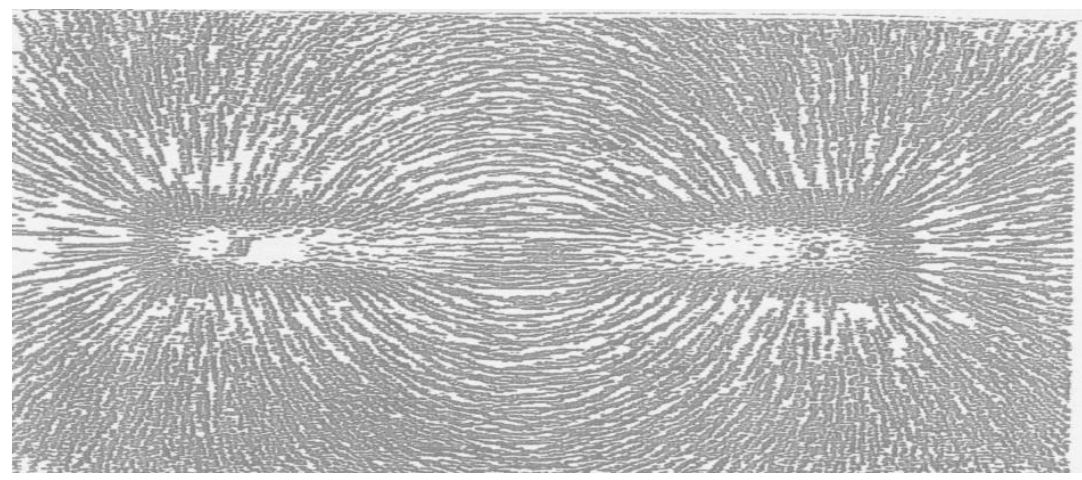

Fig2 (a): shows a bar magnet with iron filings arranged along magnetic field lines

In Figure 2a we see a bar magnet with iron filings arranged along magnetic field lines. According to Gill's electronic theory of magnetism, each magnetic field line is a combination of negative electron dependent force from the north magnetic pole and the positive proton dependent force from the south magnetic pole.

In Figure $2 \mathrm{~b}$, a wooden non-magnetic obstruction $\mathrm{Z}$ is placed on one side on the iron filings. The iron filings crumple on both sides of $\mathrm{Z}$ in zones $\mathrm{X}$ and $\mathrm{Y}$. If the magnetic force was a single force, the iron filings should have crumpled in Zone X or Zone Y only.

Thus, the magnetic force is combination of the proton dependent positive and the electron dependent negative forces from the two poles of a magnet seen both diagrammatically and experimentally.

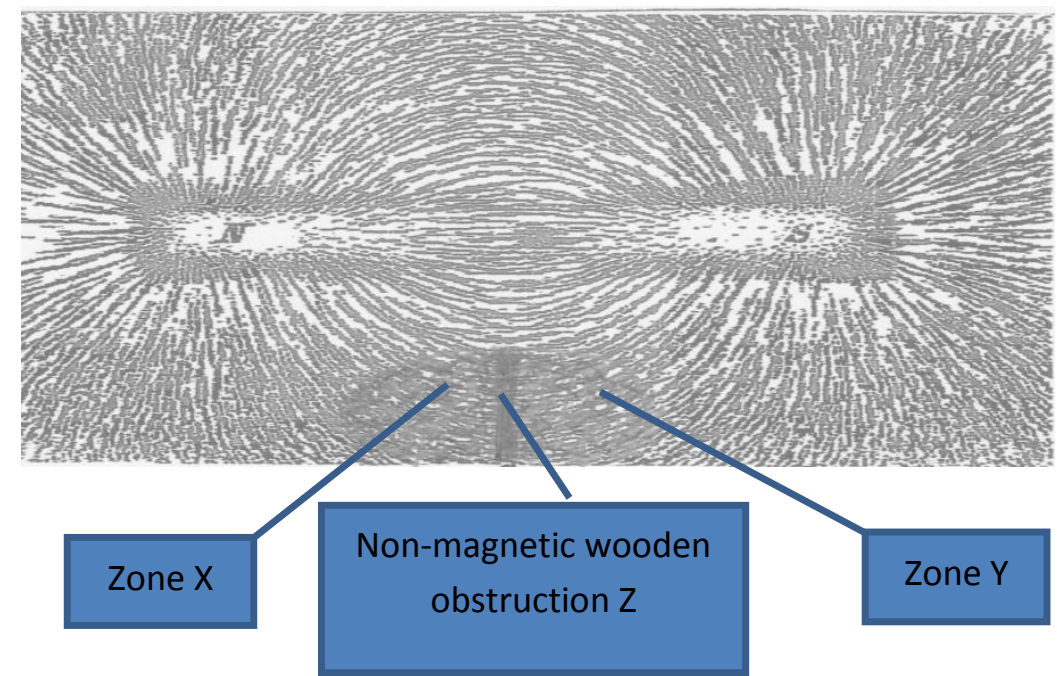

Fig2 (b): a wooden non-magnetic obstruction $Z$ is placed on one side on the iron filings

\section{Curie Point and Gill’'s Electronic Theory Of Magnetism (1964)}

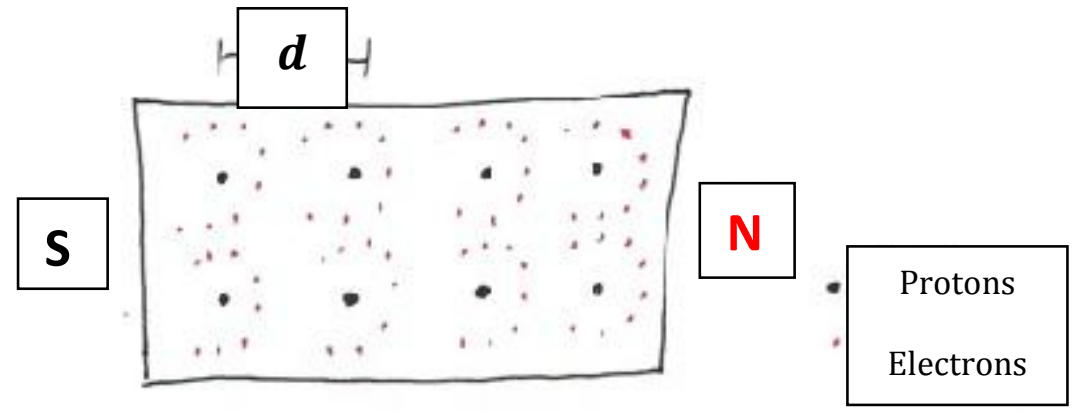

Fig3: a magnet based on Gill's electronic theory of magnetism (1964). 
Figure 3 is a magnet based on Gill's electronic theory of magnetism (1964).The inter-atomic distance between the center of one atom and the center of the next atom is $\boldsymbol{d}$ meters. In the magnetized state, some of the inner electrons with a negative clockwise torque $(-\boldsymbol{\tau})$ of an atom are able to latch onto the exposed protons with a positive anti-clockwise torque $(+\boldsymbol{\tau})$ of the next atom and so on and we have the south magnetic pole due to exposed protons with a positive anti-clockwise torque $(+\boldsymbol{\tau})$ and the north magnetic pole due to the exposed inner electrons with a negative clockwise torque $(-\boldsymbol{\tau})$ or vice versa. If the interatomic distance increases beyond a certain $\boldsymbol{d}$ meters as indicated by temperature above the Curie point or Curie temperature $\boldsymbol{T} \boldsymbol{c}$, then magnetization is not possible as the exposed inner electrons of one atom are not able to latch onto the exposed protons of the next atom.

There are two factors affecting the extent of magnetization of Figure 3:

a-the external magnetic force: The greater applied external magnetic force will result in a larger number of inner electrons latching on to the exposed protons of the next atom and so on, until we have exposed inner electrons at one end behaving as the north magnetic pole and exposed protons at the other end behaving as the south magnetic pole.

b-the inter-atomic distance: As the inter-atomic distance $\boldsymbol{d}$ meters becomes smaller with cooling, it will result in a larger number of inner electrons of one atom to link with the exposed protons of the next atom and so on until we have a larger number of exposed inner electrons at one end as the north magnetic pole and an equal larger number of exposed protons at the other end of the magnet as the south magnetic pole.

When the temperature is raised, the inter-atomic distance $\boldsymbol{d}$ meters will increase.

$$
\boldsymbol{d}=\boldsymbol{d}_{0}\left(1+\mu \cdot{ }^{\circ} K\right)
$$

where $\boldsymbol{d}_{0}$ is the inter-atomic distance at $0^{\circ} \boldsymbol{K} . \boldsymbol{K}$ is the temperature on the Kelvin scale. $\boldsymbol{\mu}$ is the coefficient of expansion of the metal.

Magnetizing force $\boldsymbol{F}$ between an inner electron $(-e)$ of one magnetized atom and the exposed proton $(+e)$ of the next magnetized atom when there is a distance $\boldsymbol{d}$ meters between them is

$$
\boldsymbol{F}=\frac{\boldsymbol{k}(+\boldsymbol{e})(-\boldsymbol{e})}{\boldsymbol{d}^{2}}=-\frac{\boldsymbol{k} \boldsymbol{e}^{2}}{\boldsymbol{d}^{2}} \text { newtons } \cdot \text { (Coulomb's law with } \boldsymbol{k} \text { as the Coulomb's constant) }
$$

Gill's electronic theory of magnetism 1964 explains that the negative force from the exposed inner electron $(-e)$ because of a clockwise negative torque $(-\tau)$ of a magnetized atom has a clockwise spin and the positive force from the exposed proton $(+e)$ because of an anti-clockwise positive torque $(+\boldsymbol{\tau})$ of the next magnetized atom has an anti-clockwise spin or vice versa depending on how you look at it. These two positive and negative forces with opposite spins will entwine with each other and attract.

As the inter-atomic distance $\boldsymbol{d}$ meters becomes larger with warming, it will result in a smaller number of inner electrons of one atom to link with the exposed protons of the next atom and so on until we have smaller number of exposed inner electrons at one end as the north magnetic pole and an equal smaller number of exposed protons at the other end of the magnet as the south magnetic pole. Curie point (Tc) is a certain high temperature at which $\boldsymbol{d}$ has increased so much that no electrons of one atom are able to latch onto the protons of the next atom and the magnet becomes de-magnetized or is unable to magnetize.

$\boldsymbol{T C}=1041^{\circ} \mathrm{K}$ for Iron.

Thus, at $\mathbf{1 0 4 1}^{\circ} \boldsymbol{K}$, the $\boldsymbol{T c}$ for iron, the inter-atomic distance $\boldsymbol{d}$ has increased so much that the inner electrons of one atom are unable to latch onto the exposed protons of the next atom and the ferromagnet is demagnetized or cannot be magnetized.

If the length of the magnet in Figure 3 is $\boldsymbol{D}$ meters and the total number of exposed electrons-e at the north magnetic pole is $\boldsymbol{n}$ which is also equal to the exposed protons+e at the south magnetic pole, then the expressed total magnetic force which will travel around the magnet (the positive force towards the electron based north magnetic pole and the negative force towards the proton based south magnetic pole) towards the two magnetic poles is

Total magnetic force $\boldsymbol{F}=\frac{\boldsymbol{k n}(+\boldsymbol{e}) \boldsymbol{n}(-\boldsymbol{e})}{D^{2}}=-\frac{\boldsymbol{k n}^{2} \boldsymbol{e}^{2}}{\boldsymbol{D}^{2}}$ newtons.

An increased external applied magnetic force $\boldsymbol{B}$ will help this latching of the inner electrons of one atom to the protons of the next atom sequentially at temperatures above the Curie point and so the Curie point can be raised by increasing the external applied magnetic force.

Curie point $(\boldsymbol{T} \boldsymbol{c})$ increase is directly proportional to the increase in external applied magnetic force $\boldsymbol{B}$.

Tc $\uparrow \infty$ external $B \uparrow$ 
As $\boldsymbol{D}$ is directly proportional to the absolute temperature $\boldsymbol{T}$

$D \propto T$

From the above discussion, we conclude that the resulting magnetizing force $\boldsymbol{F}$ is directly proportional to the external magnetizing force $\boldsymbol{B}$ and inversely proportional to the absolute temperature $\boldsymbol{T}$.

$\boldsymbol{F} \infty \boldsymbol{B}$ and $\boldsymbol{F} \infty \frac{\mathbf{1}}{\boldsymbol{T}}$ or $\boldsymbol{F} \infty \frac{\boldsymbol{B}}{\boldsymbol{T}}$ or

$F=C \cdot \frac{B}{T}$

Equation 1

Where $\boldsymbol{C}$ is a material specific constant and resulting magnetizing force $\boldsymbol{F}$ is a dot product derived with the help of Coulomb's law.

Discussion: Pierre Curie who experimented at high temperatures came up with Curie's law:

$$
M=C \cdot \frac{B}{T}
$$

Where $M$ is the resulting magnetization

$B$ is the magnetic field in teslas

$T$ is the absolute temperature in kelvins and

$C$ is a material-specific Curie constant.

According to Pierre Curie, below the Curie temperature, neighboring magnetic spins align in a ferromagnet even if there is no externally applied magnetic field. Above the Curie temperature, the magnetic spins are randomly aligned unless an external magnetic field is applied.

According to Gill's electronic theory of magnetism 1964, the exposed inner electrons of one reconfigured magnetized atom latch onto the exposed protons of the next magnetized atom and so on. Above the Curie point, due to the increased inter-atomic distance, that latching on is not possible and we have a demagnetized state. As the temperature is lowered to at or below the Curie point, the latching on and magnetization will set in and a further decrease in temperature will result in greater latching on and greater magnetization.

Equation 1 is similar to the derivation by Pierre Curie known as Curie's law except that we use dot product calculations while applying Gill's electronic theory of magnetism.

The above concept should hold good from the Curie temperature $\boldsymbol{T} \boldsymbol{c}$ all the way up to the super-cooled critical temperature for that material.

\section{WALTHER MEISSNER ANd ROBERT OCHSENFELDEXPERIMENT (1933)}

In 1933, Walther Meissner and Robert Ochsenfeld conducted an experiment shown below and came to the conclusion that the magnetic flux was expelled from inside a cooled piece of tin to the outside due to less space inside because of cooling, erroneously presuming that the total magnetic flux inside the tin cylinder and outside it was a constant independent of the temperature of the tin (Meissner effect).

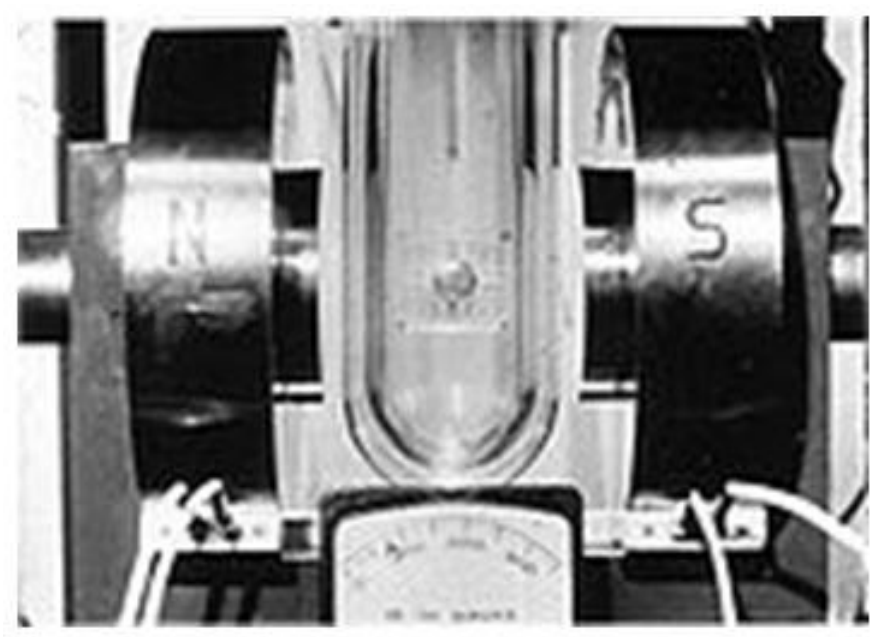


A tin cylinder-in a Dewar flask filled with liquid helium-has been placed between the poles of an electromagnet. The magnetic field is about 8 milliteslas.

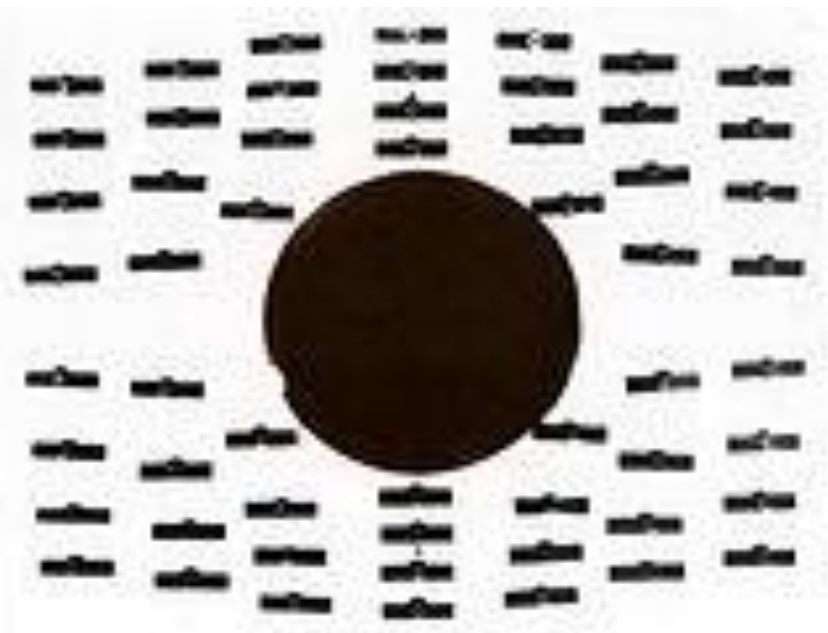

$T=4.2 \mathrm{~K}, \mathrm{~B}=8 \mathrm{mT}$. Tin is in the normally conducting state. The compass needles indicate that magnetic flux permeates the cylinder.

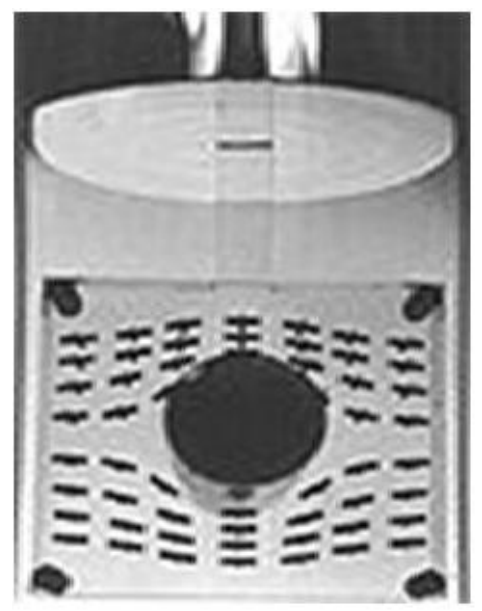

The cylinder has been cooled from $4.2 \mathrm{~K}$ to $1.6 \mathrm{~K}$. The current in the electromagnet has been kept constant, but the tin became superconducting at about $3 \mathrm{~K}$.
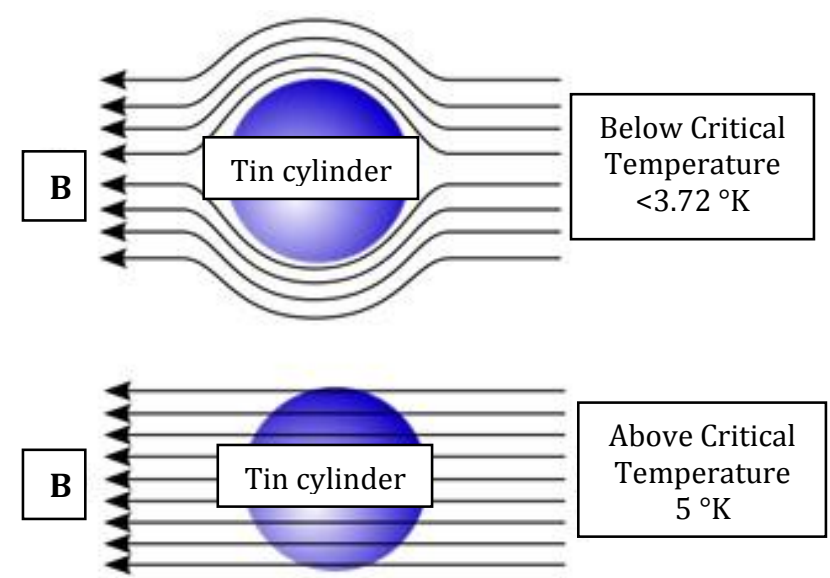

Fig4: Meissner effect due to cooling to $<3.72^{\circ} \mathrm{K}$

Figure 4 is a diagram showing the Meissner effect due to cooling to $<3.72^{\circ} \mathrm{K}$. Magnetic field lines represented as arrows, are excluded from a superconductor when it is below its critical temperature.

The Meissner effect shown above is based on the exclusion principle for lack of any other explanation. There is this constant external magnetic field $\boldsymbol{B}$ from the electromagnet applied to the tin cylinder in the 
liquid helium flask. Cooling of the tin to the critical temperature and below results in decreased interatomic space and the magnetic field lines inside the tin get expelled for lack of space.

The author disagrees with the above Meissner effect explanation and applies Gill's electronic theory of magnetism to the Meissner experiment to explain the results as follows:

\section{Line Diagrams of Meissner Experiment Plus gill’s Electronic Theory of Magnetism}

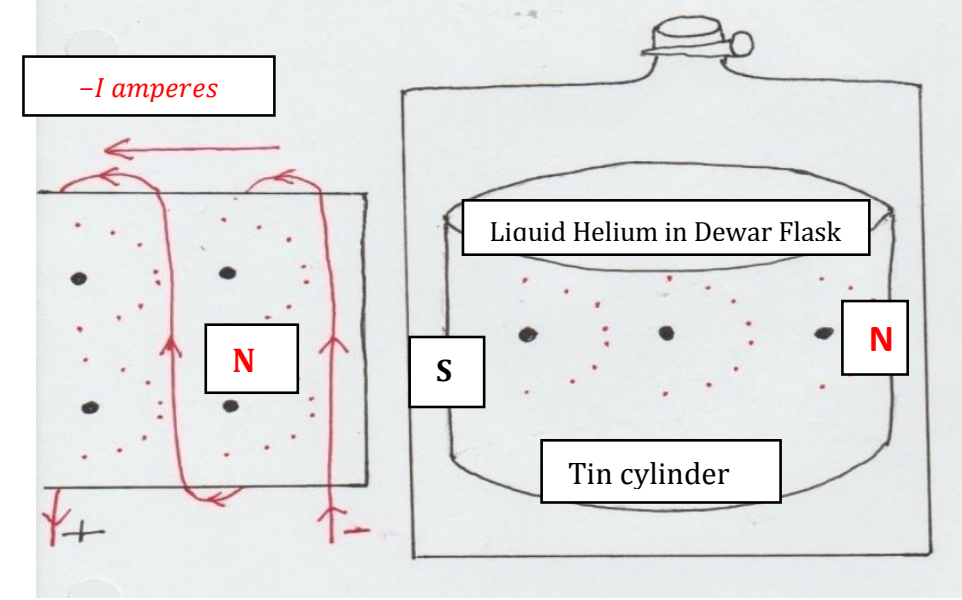

Fig5 (a): view from front

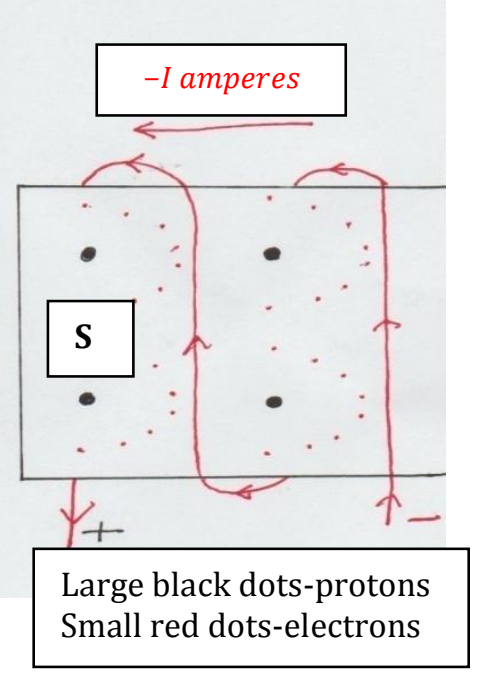

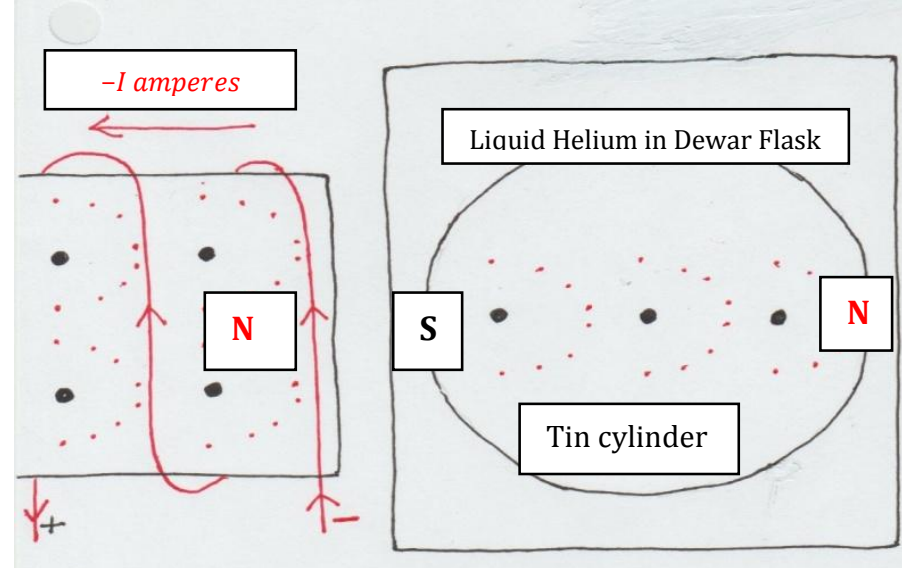

Fig5 (b): View from the top

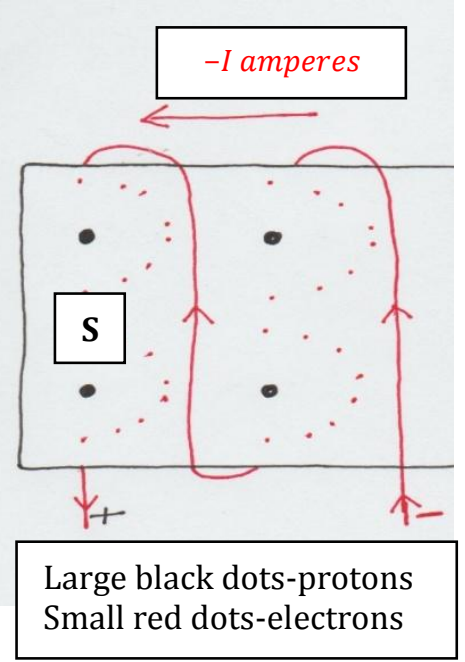

In Figure 5a and Figure 5b, we have the tin cylinder immersed in a Dewar flask with liquid helium. An electromagnet with a constant magnetic field $\boldsymbol{B}$ is placed across the Dewar flask.

The large red arrows show the overall direction of the negative electron dependent counter-clockwise electric current around the electro-magnet. According to Gill's electronic theory of magnetism, it causes each magnetized atom to have some of its inner electrons repelled towards the north magnetic pole and these electrons also develop a clockwise torque $(-\boldsymbol{\tau})$ due to repulsion from the counter-clockwise flowing electrons as an electric current. The exposed protons at the other end of each magnetized atom will have a counter-clockwise positive torque $(+\boldsymbol{\tau})$ due to the counter-clockwise force of attraction from the flowing electron dependent electric current. We thus have an electron dependent north magnetic pole (N) with a negative torque $(-\boldsymbol{\tau})$ and a proton dependent south magnetic pole $(\mathbf{S})$ with a positive torque $(+\boldsymbol{\tau})$ of the electro-magnet.

These magnetized north and south poles of the electromagnet cause a similar change in the atoms of the tin cylinder and we have a south magnetic pole in the tin towards the north magnetic pole of the electromagnet and a north magnetic pole towards the south magnetic pole of the electromagnet. The magnetic force/flux around the cylinder will be proportional to the number of exposed inner electrons at its north magnetic pole which is equal to the number of exposed protons at its south magnetic pole. The decreasing inter-atomic distance with cooling will cause greater magnetization as explained by applying 
Gill's electronic theory of magnetism which causes greater external magnetic force as seen in the above experiment. Magnetic flux cannot be expelled from inside the magnetized tin cylinder as will be explained.

\section{Intra-magnetic behavior of neighboring magnetized atoms}

When the above metal (tin) is being magnetized in a magnetic field and is cooled, the inter-atomic distance $\boldsymbol{d}$ will decrease due to cooling.

$$
d=d_{0}\left(1+\mu \cdot{ }^{\circ} K\right)
$$

$\boldsymbol{d}_{0}$ is the inter-atomic distance at $0^{\circ} \boldsymbol{K} . \boldsymbol{K}$ is temperature on the Kelvin scale.

$\boldsymbol{\mu}$ is the coefficient of expansion of the metal. $\boldsymbol{d}$ is the inter-atomic distance at a particular temperatures.

Due to the super-cooling with liquid helium, $\boldsymbol{d}$ will decrease significantly and applying Gill's electronic theory of magnetism (1964):

(a)-inner electrons of one atom will have greater ease to latch onto the protons of the next atom and so on along with an opposing torque between the electrons and the protons of each magnetized atom.

(b)-larger number of inner electrons of each atom will latch onto the protons of the next atom and so on resulting in greater magnetization.

The smaller the inter-atomic distance $\boldsymbol{d}$ which happens with lowering the temperature of the tin cylinder in the Meissner experiment, the easier it is for more of the inner electrons of one atom to latch onto the protons of the next atom and so on when subjected to the same external applied magnetic force $\boldsymbol{B}$ Also, because of the decreased $\boldsymbol{d}$ a greater number of inner electrons of each atom will latch on to the protons of the next atom and so on and result in greater magnetization of the tin cylinder without any change in the external applied magnetic $\boldsymbol{B}$ and this is what causes the Meissner effect.

\section{Intra-magnetic behavior of magnetized neighboring proton and electron}

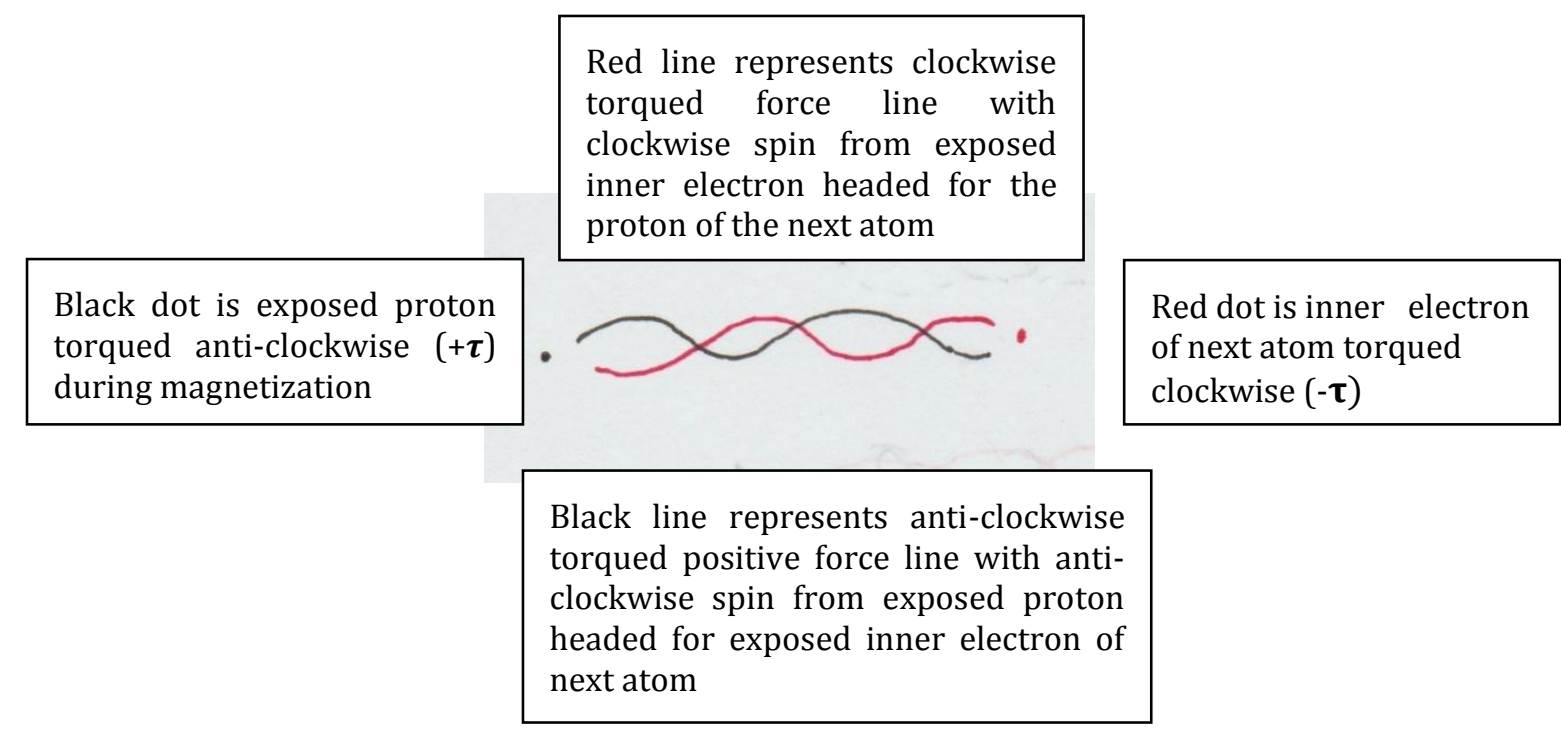

Fig6

Figure 6 is a diagrammatic representation of how the negative force from an internally exposed magnetized clockwise negatively torqued $(-\tau)$ electron $(-e)$ of an atom with a clockwise spin entwines with the positive force from an internal anti-clockwise oppositely torqued $(+\tau)$ exposed proton $(+e)$ of the next atom with an anti-clockwise spin inside the magnet. Applying Coulomb's law, the magnetizing force of attraction $\boldsymbol{F}$ between the exposed proton of an atom and the exposed inner electron of the next atom at an inter-atomic distance $\boldsymbol{d}$ meters is

$\boldsymbol{F}=\frac{\boldsymbol{k}(+\boldsymbol{e})(-\boldsymbol{e})}{\boldsymbol{d}^{2}}=-\frac{\boldsymbol{k} \boldsymbol{e}^{2}}{\boldsymbol{d}^{2}}$ newtons where $\boldsymbol{k}$ is the coulomb's constant.

If the number of magnetized atoms at each level of magnetization along the length of the magnet is $\boldsymbol{n}$ and one inner electron of one atom is connected by the opposing forces to the proton of the next magnetized atom, then the total force between the magnetized atoms at each inter-atomic level is

$\boldsymbol{F}=\frac{k n(+e) n(-e)}{d^{2}}=-\frac{k n^{2} e^{2}}{d^{2}} n e w t o n s \ldots \ldots \ldots \ldots \ldots . .$. Equation 2 
As the temperature drops during cooling, $\boldsymbol{d}$ decreases further and $\boldsymbol{n}$ increases as more electrons of each atom are able to latch onto the next atoms exposed protons, the above equation shows that this binding magnetic force $\boldsymbol{F}$ increases further and there is no possibility of this stronger binding inter-atomic intramagnetic force between electrons and protons of neighboring atoms from being expelled from inside the magnet as some Meissner effect.

\section{Extra-magnetic behavior of proton and inner electron dependent magnetic forces}
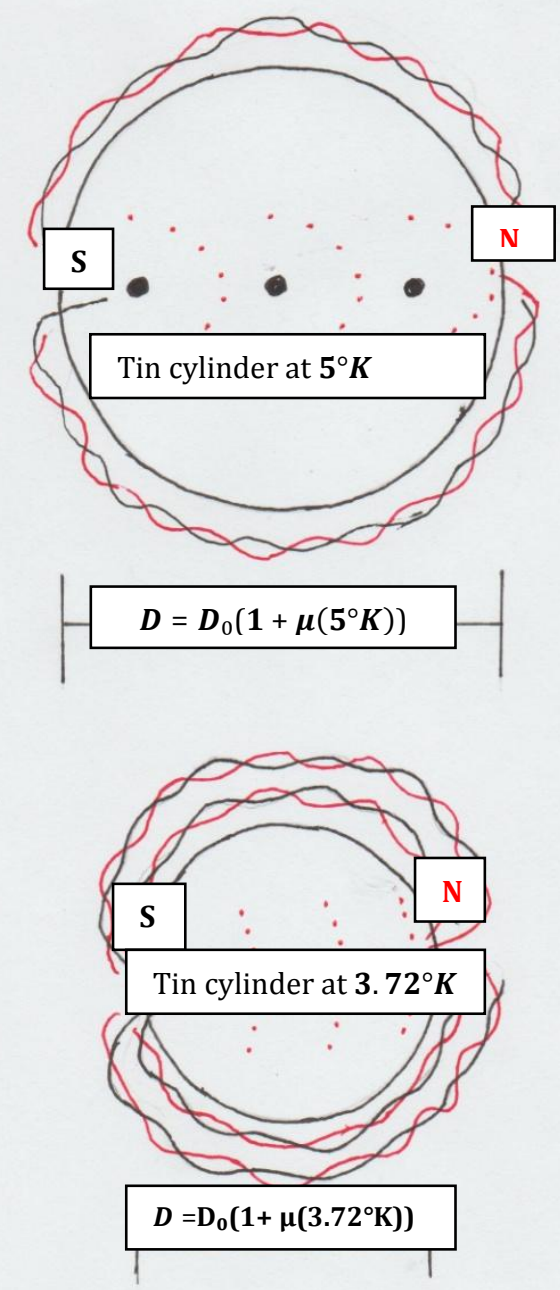

Black dots are protons

Red dots are electrons

Black lines are positive forces from protons

Red lines are negative forces from electrons

Fig7. Same tin cylinder in two stages of super-cooling at $5^{\circ} \mathrm{Kand} 3.72^{\circ} \mathrm{K}$

Figure 7 above shows the same tin cylinder in two stages of super-cooling at $5^{\circ} \mathbf{K a n d} 3 . \mathbf{7 2}^{\circ} \mathrm{K}$.

$D$ represents the diameter of the tin cylinder in meters which keeps on decreasing with cooling.

As $\mathbf{D}_{\mathbf{o}}$ meters is the diameter of the tin cylinder at absolute zero temperature of $\mathbf{0}^{\circ} \mathbf{K}$.

$D=D_{0}\left(\mathbf{1}+\boldsymbol{\mu}\left(\mathbf{5}^{\circ} \boldsymbol{K}\right)\right)$ meters at $\mathbf{5}^{\circ} \boldsymbol{K}$ and

$D=D_{0}\left(1+\boldsymbol{\mu}\left(3.72^{\circ} \mathrm{K}\right)\right)$ meters at $3.72^{\circ} \mathrm{K}$ where

$\boldsymbol{\mu}$ is the coefficient of thermal expansion of tin. The coefficient of thermal expansion of grey tin between room temperature and $-130^{\circ} \mathrm{C}$ or $143^{\circ} \mathrm{K}$ is $4.7 \times 10^{-6}$ per degree ${ }^{\circ} \mathrm{K}$ and we may use this value in the Meissner experiment.

Figure7 represents the temporary magnetization of the tin cylinder when it is placed between the two poles of an electro-magnet in the Meissner experiment. At the critical temperature of about $3.72{ }^{\circ} \mathrm{K}$, a much larger number of electrons of each atom latch onto the exposed protons of the next atom. This 
temperature drop results in an increased intra-magnetic and extra-magnetic force without any change in the applied external electro-magnetic force $\mathbf{B}$ which is constant. There is no expulsion of any magnetic flux from within the tin cylinder as the Meissner effect.

If $\boldsymbol{n}$ is the number of exposed inner electrons $(-\boldsymbol{e})$ with a negativetorque $(-\boldsymbol{\tau})$ as the north magnetic pole and an equal number of exposed protons $(+\boldsymbol{e})$ with a positive torque $(+\boldsymbol{\tau})$ as the south magnetic pole, then the external magnetic force spreads around the tin cylinder half circumference between the two magnetic poles $\left(\pi \cdot \frac{D}{2}\right.$ meters $)$.

As these two magnetic poles are actually at a direct distance $\boldsymbol{D}=\boldsymbol{D}_{0}\left(\mathbf{1}+\boldsymbol{\mu}^{\circ} \boldsymbol{K}\right)$, applying Coulomb's law, the external magnetizing force around the tin cylinder is

$$
\begin{gathered}
\boldsymbol{F}=\frac{\boldsymbol{k n}(-\boldsymbol{e}) \boldsymbol{n}(+\boldsymbol{e})}{D^{2}}=\frac{\boldsymbol{k n}(-\boldsymbol{e}) \boldsymbol{n}(+\boldsymbol{e})}{\left(D_{0}\left(\mathbf{1}+\boldsymbol{\mu}^{\circ} \boldsymbol{K}\right)\right)^{2}} \text { newtons }=-\frac{\boldsymbol{k n}^{2} \boldsymbol{e}^{2}}{\left(D_{0}\left(\mathbf{1}+\boldsymbol{\mu}^{\circ} \boldsymbol{K}\right)\right)^{2}} \text { newtons } \\
1 \boldsymbol{e} \approx \mathbf{1 . 6} \times \mathbf{1 0}^{-19} \text { Coulombs }
\end{gathered}
$$

Coulombs constant $\boldsymbol{k}=\mathbf{8 . 9 9} \times 10^{9} \mathrm{Nm}^{2} \mathrm{C}^{-2}$.

$\boldsymbol{\mu}$ is the coefficient of thermal expansion for tinis4.7 $\times \mathbf{1 0}^{-6}$ per $\operatorname{degree}^{\circ} \mathrm{K}$.

${ }^{\circ} \boldsymbol{K}$ represents the absolute temperature on the kelvin scale and

$\boldsymbol{D}=\boldsymbol{D}_{0}\left(\mathbf{1}+\boldsymbol{\mu}^{\circ} \boldsymbol{K}\right)$ is the diameter of the tin cylinder at that temperature.

Thus, the flux/magnetic force seen around the tin cylinder is directly proportional to the square of number of exposed protons at one end, which is also equal to the square of the number of exposed inner electrons at the other end $\left(\boldsymbol{n}^{2}\right)$ and is inversely proportional to the square of the diameter of the tin cylinder $\left(\boldsymbol{D}^{2}\right)$.

$\boldsymbol{F} \propto \boldsymbol{n}^{2}$ and $\boldsymbol{F} \propto \frac{1}{\left(D_{0}\left(1+\mu^{\circ} K\right)\right)^{2}}$

As the absolute temperature ${ }^{\circ} \boldsymbol{K}$ decreases, the external magnetizing force $\boldsymbol{F}$ increases.

The same tin cylinder when cooled to $3.72^{\circ} \mathrm{K}$ which is the critical temperature, a maximal number of the inner electrons of each magnetized atom latch onto the exposed protons of the next atom and so on and we have an increased magnetic force from the two magnetic poles of the tin cylinder manifesting around the tin cylinder.

The external magnetizing force $\boldsymbol{F}=\frac{\boldsymbol{k n}(+\boldsymbol{e}) \boldsymbol{n}(-\boldsymbol{e})}{\left(\boldsymbol{D}_{0}\left(\mathbf{1}+\boldsymbol{\mu}^{\circ} \boldsymbol{K}\right)\right)^{2}}=-\frac{\boldsymbol{k n}^{2} \boldsymbol{e}^{2}}{\left(\mathbf{D}_{0}\left(\mathbf{1}+\boldsymbol{\mu}^{\circ} \mathbf{K}\right)\right)^{2}}$ newtons .............Equation 3

With decreasing temperature, the resultant diameter of the tin cylinder $\boldsymbol{D}=\boldsymbol{D}_{0}\left(\mathbf{1}+\boldsymbol{\mu}^{\circ} \boldsymbol{K}\right)$ keeps on decreasing resulting in an increase in the external magnetizing force

As $\boldsymbol{D}$ is much larger than $\boldsymbol{d}$, Equation 3 is a small fraction of Equation 2, but, the larger intra-magnetic binding force is used up for binding the electrons and protons of the neighboring magnetized atoms. However, Equation 3 is enough to explain the increase in external magnetic force on cooling as seen in the Meissner experiment in 1933 and there is no need to resort to the expulsion of magnetic flux to explain the Meissner effect.

The decreased temperature also causes a decreased inter-atomic distance $\mathbf{d}$ which causes an increased number of electrons of one magnetized atom to latch onto the exposed protons of the next magnetized atom resulting in an increased $\mathbf{n}$ which causes an increased external magnetic force at the critical temperature on the outside of the tin cylinder without any expulsion of the intra-magnetic force called the Meissner effect.

At the critical temperature the inter-atomic distance $\boldsymbol{d}$ has decreased significantly and Equation 2 shows that the binding force between the exposed inner electrons of one magnetized atom and the exposed protons of the next magnetized atom is maximal and this stronger internal magnetic force has been used as a binding force and cannot be expelled or squeezed out as a Meissner effect.

Please note that $\boldsymbol{n}$ depends on:

-the external applied magnetic force $\boldsymbol{B}$ (which is a constant in the Meissner experiment) and

- the inter-atomic distance inside the tin cylinder which depends on the temperature of the tin cylinder. 
Thus, we will have a greater number of exposed electrons at one end of the tin piece torqued in clockwise direction $(-\boldsymbol{\tau})$ manifesting as a clockwise spinning negative force and an equal greater number of exposed protons at the other end of the tin piece torqued in the anti-clockwise direction $(+\boldsymbol{\tau})$ manifesting as an anti-clockwise spinning positive force.

This results in a greater magnetic force effect on the outside as shown in Figure 7, without any magnetic flux being pushed out along the length of the super-cooled tin cylinder at the critical temperature.

\section{SUPERCONDUCTIVITY}

Superconductivity is a phenomenon of zero electrical resistance occurring in certain materials called superconductors, when cooled below a characteristic critical temperature. It was discovered by Dutch physicist Heike Kamerlingh Onnes on April 8, 1911, in Leiden. In a superconductor, the resistance drops abruptly to zero when the material is cooled below its critical temperature. An electric current through a loop of superconducting wire can persist indefinitely with no power source.

Superconductivity will result when due to super-cooling below the critical temperature, the inter-atomic distance of the conductor decreases so that adjacent atoms merge with each other. In this situation, the free valence electrons are almost equidistant from the protons of neighboring atoms and will flow with zero resistance for an indefinite period.

The inter-atomic distance $d$ at a temperature on the Kelvin scale is given by

$$
d=d_{0}\left(1+\mu^{\circ} K\right)
$$

$\boldsymbol{d}_{\mathbf{0}}$ is the inter-atomic distance at $\mathbf{0}^{\circ} \boldsymbol{K} .{ }^{\circ} \boldsymbol{K}$ is temperature in Kelvins. $\boldsymbol{\mu}$ is the coefficient of expansion of the conducting material.

On increasing the temperature of a wire, the inter-atomic distance $\boldsymbol{d}$ increases and there is greater resistance to the flow of outer free electrons or electric current.

Electrical resistance $\boldsymbol{R}$ in Ohms is directly proportional to the absolute temperature ${ }^{\circ} \boldsymbol{K}$ on the Kelvin scale.

$$
\text { Resistance } \boldsymbol{R}\left(\text { Ohms) } \infty \text { Temperature }{ }^{\circ} \boldsymbol{K}\right. \text { (Kelvin scale) }
$$

Cooling the above wire will decrease the inter-atomic distance $\boldsymbol{d}$ and in a super cooled state, there will be no inter-atomic gap and in this situation, there will be zero resistance to the flow of the outer free electrons(electric current) and at or below a critical temperature, we have a super-conducting wire. At this critical temperature, the outer free valence electrons are almost equidistant from the protons of the neighboring atoms due to an absent gap between the atoms and will be able to move with zero resistance across atoms and we will have a superconductivity state.

Superconductivity is inversely proportional to the absolute temperature on the Kelvin scale.

$$
\text { Superconductivity } \infty \frac{1}{{ }^{\circ} \mathrm{K}}
$$

In electromagnetism, magnetic susceptibility $\chi$ is aproportionality constant that indicates the degree of magnetization of a material in response to an applied magnetic field.

Magnetic susceptibility $\boldsymbol{\chi} \infty$ applied magnetic field $\boldsymbol{B}$

Magnetic susceptibility $\chi \infty \frac{\mathbf{1}}{{ }^{\circ} \boldsymbol{K}}$.

Combining these two we have

Magnetic susceptibility $\boldsymbol{\chi} \infty \frac{\boldsymbol{B}}{{ }^{\circ} \boldsymbol{K}}$ or

Magnetic susceptibility $\chi=C \frac{\boldsymbol{B}}{{ }^{\circ} \boldsymbol{K}}$

where $\boldsymbol{C}$ is a material specific constant.

From the above discussion, based on Gill's electronic theory of magnetism, we conclude that magnetic susceptibility is directly proportional to the applied magnetic field and it is inversely proportional to the absolute temperature on the Kelvin scale. 


\section{DISCUSSION}

Gill's electronic theory of magnetism explains how the magnetic strength in a material can vary with temperature when everything else is a constant.

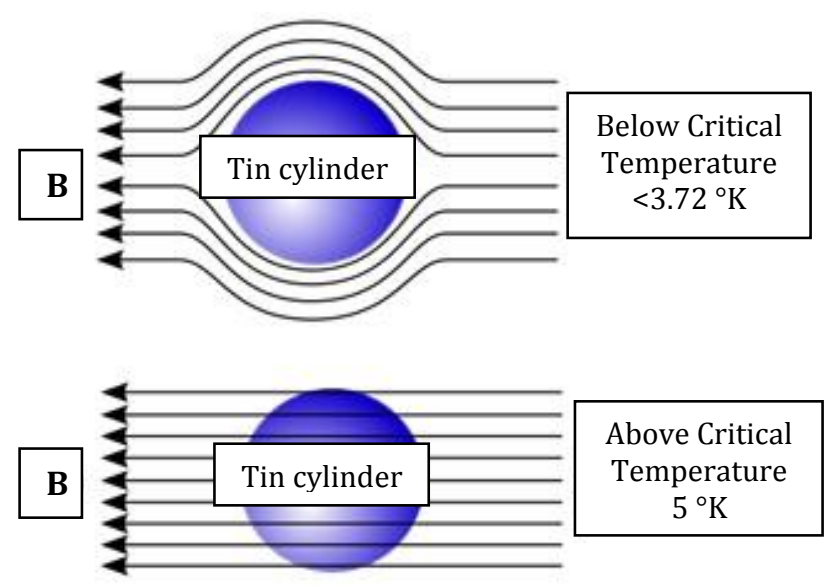

Above is a repeat diagram of Figure 4 showing the Meissner effect due to cooling to $<3.72^{\circ} \mathrm{K}$. Magnetic field lines, represented as arrows, are excluded from a superconductor when it is below its critical temperature.

Meissner applied the conservation of magnetic flux concept by a superconductor and came to the conclusion that when the interior field decreases, the exterior field increases and called it the Meissner effect.

The notion of conservation of magnetic flux to explain the increased magnetic flux by expulsion of internal magnetic flux below a critical superconducting temperature as shown in Figure 4 is wrong. A superconductor with little or no magnetic field within it is said to be in the Meissner state is not a correct concept.

This conservation of magnetic flux without considering the change in the intra-magnetic and outer or extra-magnetic forces due to change in inter-atomic distance of the tin atoms caused by temperature change and its effect on the magnetic force is a mistake.

It has been explained that the even stronger intra-magnetic binding forces due to cooling as calculated by Equation 2 cannot be expelled as a magnetic flux and there is no Meissner effect. The increase in external magnetic force outside the tin cylinder is due to cooling of the tin cylinder with liquid helium in the Meissner experiment (1933)as calculated by Equation 3and there is no expulsion of magnetic flux from within the tin cylinder to without as a Meissner effect.

Applying Gill's electronic theory of magnetism, the atoms of the tin cylinder have undergone a temporary magnet like change in configuration due to the effect of the external applied electromagnetic force. Thus, just by lowering the temperature, we have increased internal magnetic forces in a sequential manner as explained above which are even more strongly merged with neighboring magnetized atoms and cannot be expelled and the tin cylinder undergoes greater magnetization due to reduced inter-atomic distance manifested at its magnetic ends. It is this greater magnetization which is seen as an increased external magnetic effect or Meissner Effect.

The levitation of the electron dependent north magnetic pole of a magnet above the super-cooled tin cylinder is because of the dense layer of electrons on the surface of the tin cylinder cooled to the critical temperature.

\section{STAGES OF MAGNETIZATION}

Combining Pierre Curies high temperature experiments with the Walther Meissner and Robert Ochsenfeld low temperature experiment (1933) and applying Gill's electronic theory of magnetism (1964) to both of them, we could have the following stages of magnetism:

In below Figure 8, we have the same metal showing differing magnetic behavior at different temperatures with the same external applied magnetic force B according to Gill's electronic theory of magnetism. 


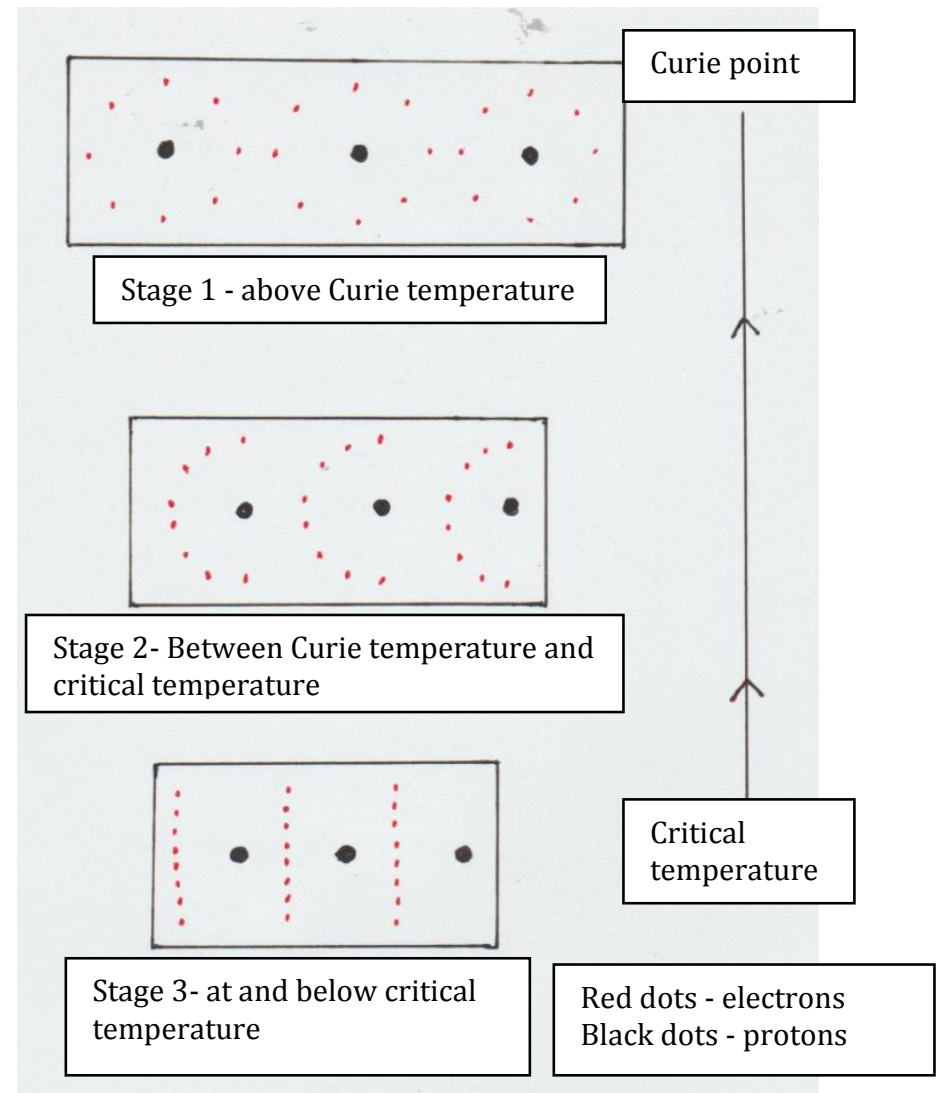

Fig8. Same metal showing differing magnetic behavior at different temperatures with the same external applied magnetic force B according to Gill's electronic theory of magnetism.

Stage1: This is at and above the Curie temperature and the neighboring atoms due to the increased interatomic distance are not able to latch onto one another to behave as a magnet.

Stage 2: Between the Curie temperature and the critical temperature for that material, the exposed inner electrons of a magnetized atom are able to latch onto the exposed protons of the next atom. There is also an increasing magnetization along with reduction of electrical resistance as the inter-atomic distance keeps on decreasing with decreasing temperature.

Stage 3: At the critical temperature for the material, a larger number of inner electrons of one atom are able to latch onto the exposed protons of the next atom, as there is no gap between the atoms due to the low temperature. In a super-cooled state, when the atoms of that material have no or minimal gap between them, the outer free valence electrons will flow as an electrical current against zero resistance once started as an electrical flow. The outer free valence electrons are able to move across these atoms which have no or minimal gap as an indefinite electric current once started due to zero resistance and we have the super-conducting state

According to Gill's electronic theory of magnetism (1964), the increased magnetic force observed outside the tin cylinder is due to the increased magnetization of the tin due to decreased inter-atomic distance due to lowering of the temperature. Even though the external applied magnetic field $\boldsymbol{B}$ is the same, due to the decreased inter-atomic distance $\boldsymbol{d}$, a larger number of inner electrons of a magnetized atom of tin latch onto the next magnetized atom sequentially and we have a larger number of exposed inner electrons at the north magnetic pole of the magnetized tin and a larger number of exposed protons at the south magnetic pole of the tin piece. Thus, we have an increased magnetic force outside the tin piece just by cooling without any change in the applied external magnetic field $\boldsymbol{B}$ and without any expulsion of the intra-magnetic force from the magnetized tin.

The London equations developed by brothers Frietz and Heinz London in 1935 offered the simplest meaningful description of superconducting phenomena by trying to explain the actually non-existent Meissner effect.

It has been explained in this article that a material does not exponentially expel any internal magnetic fields or magnetic flux as it has been called as it crosses the superconducting threshold. Rather, a decrease 
in the inter-atomic distance $\boldsymbol{d}$ will prevent any magnetic flux from being expelled to the outside. There might be minimal surface activity as explained by Andre Marie Ampere in 1823 but no expulsion or squeezing out of magnetic flux or Meissner effect.

\section{CONCLUSION}

Gill's electronic theory of magnetism has helped in explaining that the Meissner effect is because of increased external magnetic force around the tin cylinder on cooling. Thisis due to the increased magnetization due to the decreased temperature causing the decreased inter-atomic distance in the tin cylinder and there is no expulsion or squeezing out of magnetic flux (Meissner effect)in the 1933 Meissner experiment as previously postulated.

Curie point and its variability because of a change in the external applied magnetic force and/or temperature has been explained with Gill's electronic theory of magnetism along with some dot product equations.

Superconductivity and levitation of a magnet have been discussed. The relation between electric current and electromagnetic fields around a superconductor has been explained with the help of Gill's electronic theory of magnetism.

Gill's electronic theory of magnetism (1964) explains the interaction between the magnetic forces and the electrical forces and does away with the asymmetry issue raised by A. Einstein in 1905 and Richard Feinmann in 1943.

\section{Acknowledgment}

I am grateful to physicist Professor Amitabha Mukherjee (former head of department of Physics, Delhi University) for going over this article with me.

\section{REFERENCES}

1. Faraday's iron ring experiment (1831) explained with Gill's electronic theory of magnetism (1964) by author ARJPS 2017

2. A Treatise on Electricity and Magnetism by James Clerk Maxwell (1851).

3. Ampère's circuital law by Andre Marie Ampere (1823)

4. Theoretical remark on the superconductivity of metals by A. Einstein

5. Thermal Expansion of Grey Tin by J.Thewlis \& A.R.Davley

6. Large Increase of the Curie Temperature by Aymeric Sadoc, Bernard Mercey, Charles Simon, Dominique Grebille, Wilfrid Prellier, and Marie-Bernadette Lepetit

7. Pierre Curie-Biography Nobelprize.org. Nobel Media AB. 2014. Retrieved 14 March 2013.

8. The Theory of Superconductivity, Nikolaŭ Nikolaevich Bogoliubov (1963)

9. The Discovery of Superconductivity Dirk van Delft and Peter Kas

10. Meissner effect from Wikipedia

11. Coulomb's law by Charles Augustin de Coulomb (1784)

Citation: Avtar Singh Gill. "Meissner Effect (1933) Re-Explained by Gill's Electronic Theory of Magnetism (1964)", American Research Journal of Physics, vol 4, no. 1, 2018, pp. 1-14.

Copyright (C) 2018 Avtar Singh Gill. This is an open access article distributed under the Creative Commons Attribution License, which permits unrestricted use, distribution, and reproduction in any medium, provided the original work is properly cited. 